\title{
Abordaje de la vía aérea en el paciente neuroquirúrgico
}

\section{Airway approach in the neurosurgical patient

\author{
Dra. Claudia Sánchez-Torres,* Dra. Cecilia Úrsula Mendoza-Popoca,* \\ Dr. Mario Suárez-Morales,* Dr. Joel Rodríguez-Reyes ${ }^{\ddagger}$
}

Citar como: Sánchez-Torres C, Mendoza-Popoca Cú, Suárez-Morales M, Rodríguez-Reyes J. Abordaje de la vía aérea en el paciente neuroquirúrgico. Rev Mex Anestesiol. 2021; 44 (4): 272-276. https://dx.doi.org/10.35366/100872

RESUMEN. El abordaje de la vía aérea en el paciente neuroquirúrgico presenta grandes retos debido al escenario tan complejo al cual nos enfrentamos; debemos considerar las características propias del paciente, las comorbilidades presentes y la patología neurológica por la que va a ser intervenido. Conocer la patología neurológica y las implicaciones o repercusiones que ésta puede llegar a tener en el manejo de la vía aérea ayudarán a la toma de decisiones y conocer los retos y escenarios que se pudieran presentar durante el evento anestésico-quirúrgico.

ABSTRACT. The approach to the airway in the neurosurgical patient presents great challenges due to the complex scenario we face; we must consider the patient's own characteristics, the co-morbidities present and the neurological pathology for which it is going to be intervened. Knowing the neurological pathology and the implications or repercussions that this may have over the management of the airway will help decision making and manage the challenges and scenarios that could arise during the anesthetic surgical event.

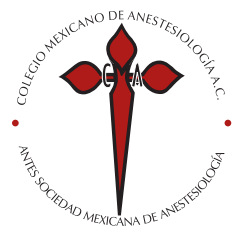

Palabras clave:

Craneotomía, craneotomía despierto, trauma medular, vía aérea.

Keywords:

Craniotomy, awake craniotomy, spinal trauma, airway.

\footnotetext{
* Neuroanestesiología.

‡ Anestesiología, Medicina del

Enfermo en Estado Crítico.
}

Centro Médico ABC.

Correspondencia:

Dra. Claudia Sánchez-Torres Centro Médico ABC, Campus Observatorio Sur 136 No. 116, Col. Las Américas, Alcaldía Álvaro Obregón, 01120, CDMX. Teléfono: 3316002270

E-mail: claudia_sancheztor@

hotmail.com

Recibido: 09-10-2019

Aceptado: 15-07-2020

\section{INTRODUCCIÓN}

$\mathrm{E}$ l abordaje de la vía aérea requiere de habilidades técnicas, pero también de las llamadas habilidades no técnicas que permitan reconocer el contexto del paciente y así poder resolver los problemas que se pueden llegar a presentar durante su intervención ${ }^{(1)}$.

Uno de los objetivos primarios en el abordaje de la vía aérea es poder establecer en cualquier paciente una ventilación y oxigenación adecuadas ${ }^{(2)}$. El manejo de la vía aérea en el paciente neuroquirúrgico no difiere mucho de los estándares de manejo recomendados en las guías internacionales; sin embargo, en éstas sólo se toman en cuenta las necesidades de la anestesia general para todo tipo de procedimiento quirúrgico, pero no consideran las grandes diferencias directamente relacionadas con la naturaleza de algunos procedimientos neurológicos y sus implicaciones clínicas, las cuales presentan retos específicos para mantener la ventilación y la vía aérea permeable en el proceso perioperatorio ${ }^{(3)}$.
Las estadísticas reportadas en las guías DAS (Difficult Airway Society) 2015 demuestran que no existe ningún sistema que pronostique en forma confiable, la dificultad para el abordaje de la vía aérea, pero sí se ha evidenciado que una deficiente evaluación preoperatoria, ya sea por omisión, evaluación incompleta o error en la modificación del manejo, secundario a los hallazgos de la evaluación, son factores para el desenlace negativo en todo procedimiento ${ }^{(4)}$.

La vía aérea difícil constituye un problema de salud real que, a pesar de la mejora en las herramientas para su abordaje, requiere una evaluación exhaustiva ${ }^{(4)}$.

El manejo de la vía aérea en los pacientes neuroquirúrgicos implica retos únicos condicionados por las características propias del paciente, la patología neurológica y el área en la cual se realizará el procedimiento. La gran variedad de particularidades en procedimientos neuroquirúrgicos tales como posiciones especiales (sedente, concorde, park bench, entre otras) y la complejidad de los instrumentos usados para su 
intervención, representan obstáculos que impiden el acceso a la cabeza y a la vía aérea del paciente, por ello es primordial planificar cuidadosamente la mejor estrategia para el manejo de la vía aérea durante todo el procedimiento, ya que es parte del abordaje de la vía aérea (Figura 1).

Se debe tener en consideración que la patología neurológica va estrechamente relacionada con la selección de la técnica más segura para el manejo de la vía aérea, ya que algunos de los pacientes tienen alterado el estado de despierto o algún grado de deficiencia neurológica, lo que se ve reflejado en la disminución de reflejos protectores de la vía aérea o en el control de la ventilación ${ }^{(5,6)}$.

La valoración, planeación y prevención de catástrofes en cada procedimiento tienen como beneficio no sólo la segu- ridad de éste, sino también la optimización de tiempos en el perioperatorio.

Por lo cual, a continuación se describen los diferentes escenarios de manejo de vía aérea en el paciente neuroquirúrgico.

\section{MANEJO DE LA VÍA AÉREA EN PACIENTE CON TRAUMA MEDULAR CERVICAL}

Las lesiones de la columna cervical representan alrededor de 2-3\% de las lesiones en los pacientes víctimas de traumatismo cerrado. De todas las lesiones de columna, entre 19 y $51 \%$ se localizan en la región cervical; esto tiene un gran impacto, ya que se considera que es la patología con mayor incidencia de lesión medular y mortalidad(7).

Figura 1:

Importancia del correcto posicionamiento del paciente para cirugía neurológica.
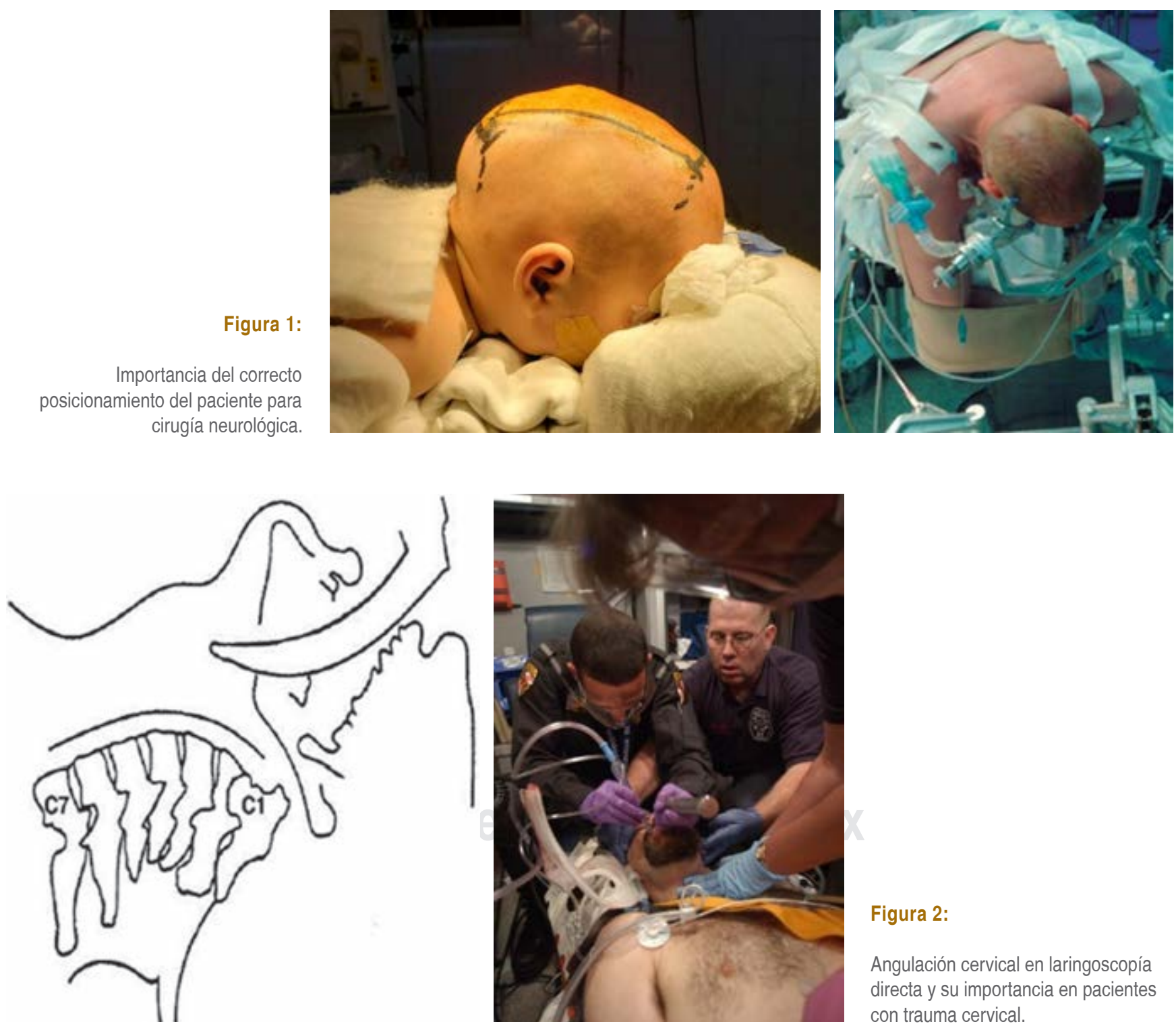

Figura 2:

Angulación cervical en laringoscopía directa y su importancia en pacientes con trauma cervical. 

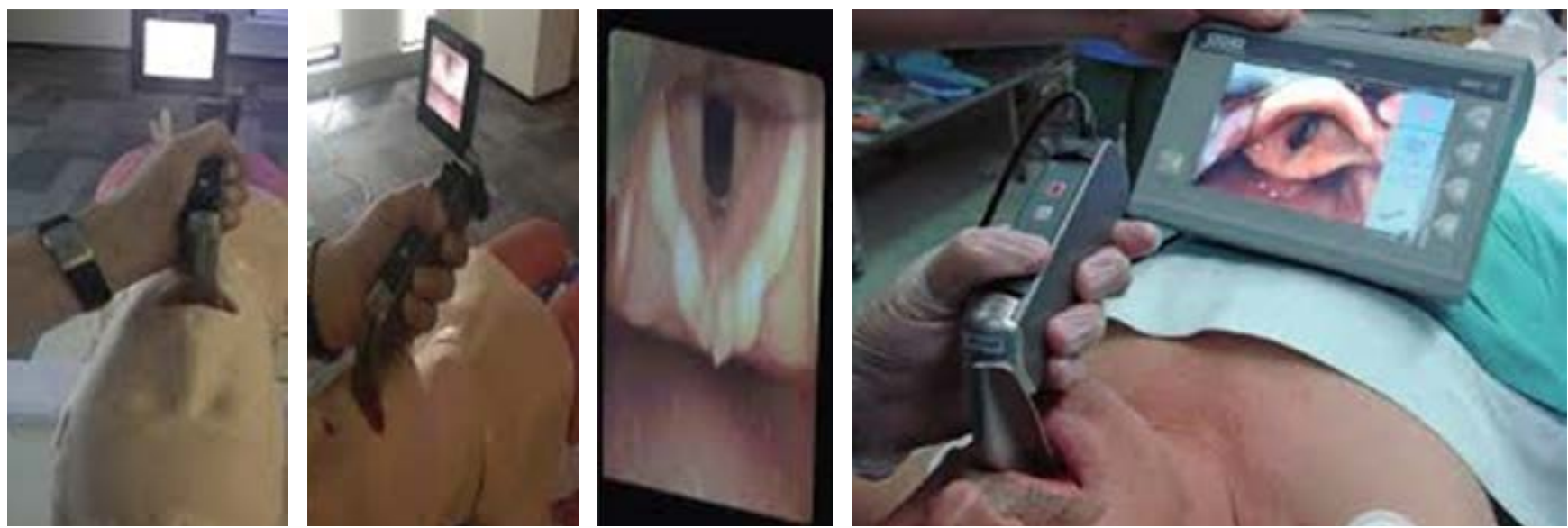

Figura 3: Visualización glótica mediante videolaringoscopía.
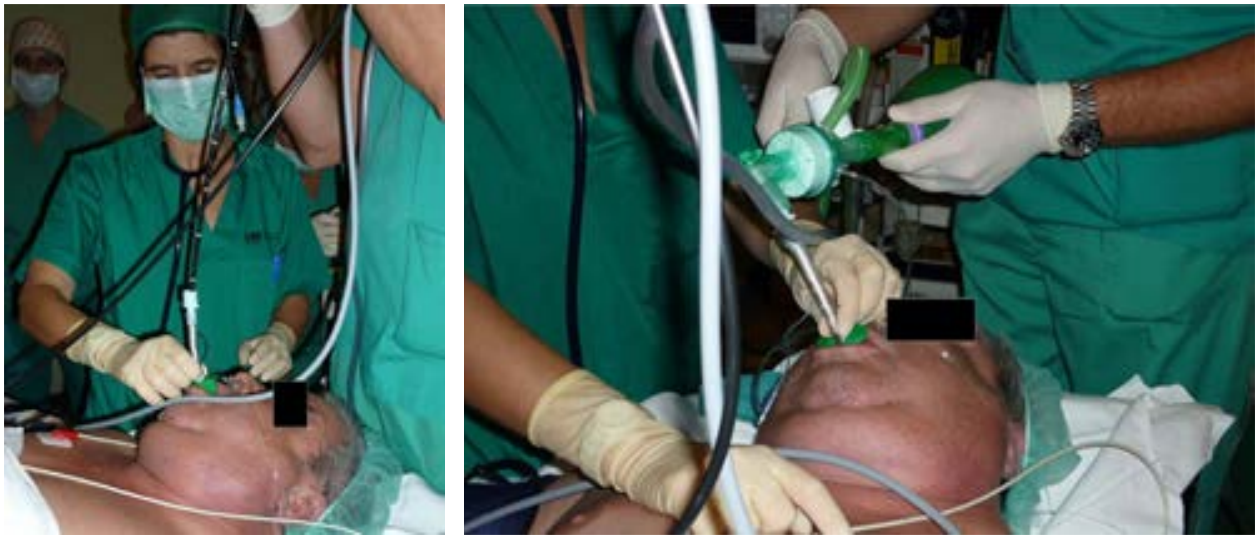

Figura 4:

Uso de fibrobroncoscopio en paciente con vía aérea difícil.

El objetivo primario en el manejo de la vía aérea de los pacientes con lesión medular cervical es minimizar el movimiento del cuello y conseguir un rápido y eficaz aseguramiento de la misma.

El National Emergency X-Radiography Utilization Study (NEXUS) diseñó el Low-Risk Criteria (NLC) para identificar los cinco criterios clínicos que identificarán a los pacientes de bajo riesgo y que incluyen: no presentar dolor cervical en la línea media, ausencia de déficit neurológico focal, estado de alerta normal, ausencia de intoxicación y ninguna lesión dolorosa (Tabla 1) ${ }^{(8)}$.

Todas las maniobras básicas para inmovilizar la vía área tienen efecto en menor o mayor grado sobre la lesión cervical ${ }^{(9)}$. El gran reto en los pacientes con trauma cervical radica en la limitación para la ventilación, secundaria al tipo de dispositivos que se emplean para la inmovilización del cuello. Maniobras como la tracción mandibular que faciliten la apertura de la vía aérea causan desplazamiento de la columna cervical, lo cual ha sido observado en modelos cadavéricos; con lo que se comprueba que al momento de la laringoscopía se pueden producir lesiones secundarias sólo por la manipulación ${ }^{(10)}$.
La laringoscopía directa produce una gran movilidad en la unión craneocervical con moderada movilización de C1-C2 y mínimo desplazamiento de C4 en pacientes sanos, por lo que no está indicada en pacientes con lesión o sospecha de lesión cervical (Figura 2).

La inmovilización manual cráneo-cervical en línea es la forma más frecuente de inmovilización durante la manipulación de la vía aérea, ya que limita los movimientos del cuello.

Durante el uso de la maniobra de inmovilización manual se ha reportado aumento en la tasa de fracaso de intubación en algunos pacientes, durante los primeros 30 segundos. Si se compara con la inmovilización con collarín, la inmovilización manual en línea reduce el movimiento de la columna, por lo que se recomienda cuando se sospecha de lesión cervical(11).

Los pacientes con lesión cervical se encuentran en riesgo de obstrucción de la vía aérea, por lo que el manejo de ésta se convierte en una prioridad.

La videolaringoscopía es una técnica que aumenta el éxito de intubación; también mejora la visualización glótica, me- 
dida por la clasificación de Cormack-Lehane; además, se ha demostrado que, aunada a la inmovilización manual en línea, disminuye de manera significativa el riesgo de daño cervical durante la intubación (Figura 3)(12).

La utilización del fibrobroncoscopio flexible reduce notablemente la movilización cervical, pero se requiere de adiestramiento especial para su uso. La mascarilla laríngea ha demostrado ser el dispositivo que causa mayor desplazamiento posterior de la columna cervical, tanto durante su inserción como una vez colocada (Figura 4$)^{(9)}$.

En conclusión, no existe un modo perfecto de manejar la vía aérea en los pacientes con potencial lesión medular cervical; sin embargo, se debe tener en consideración los posibles riesgos de cada uno de los instrumentos (Tabla 2) ${ }^{(9)}$.

El manejo de la vía aérea debe individualizarse tomando en consideración las características del paciente, la intervención a realizar, si se trata o no de una urgencia y el nivel de experiencia de la persona que tiene el cargo de manipular la vía aérea. El objetivo principal es evitar una lesión secundaria ${ }^{(13)}$.

\section{Tabla 1: Los criterios NEXUS de bajo riesgo.}

Criterios para bajo riesgo de lesión cervical:

1. Ausencia de sensibilidad en la línea media.

2. Ausencia de focalidad neurológica.

3. Nivel de alerta normal.

4. Ausencia de lesión dolorosa que distraiga al paciente de dolor localizado en la columna.

5. Sin evidencia de intoxicación.

Modificada de: Galeiras VR, Ferreiro VME, Mourelo FM, Montoto MA, Salvador de la Barrera S. Actualización en lesión medular aguda postraumática. Parte 1. Medicina Intensiva. 2017:41:237-247. Disponible en: https://doi.org/10.1016/j.medin.2016.11.002

\section{MANEJO DE LA VÍA AÉREA EN CRANEOTOMÍA}

La colocación quirúrgica del paciente programado para una craneotomía es un escenario realmente desafiante. Agregado a lo anterior, la fijación de la cabeza a la mesa quirúrgica se lleva a cabo mediante el uso del cabezal de Mayfield; esto requiere de una manipulación peligrosa del cuello que puede comprometer la permeabilidad de la vía aérea ${ }^{(3)}$.

Los pacientes sin criterios de una vía aérea difícil que requieren intubación orotraqueal son abordados mediante laringoscopía directa. En pacientes con vía aérea difícil predicha, el uso de videolaringoscopio o fibrobroncoscopio flexible debe anticiparse ${ }^{(14)}$.

Para asegurar la vía aérea es recomendable el uso de sonda endotraqueal armada cuando la cabeza no se coloca en posición neutral, para disminuir así el riesgo de obstrucción del tubo orotraqueal por acodamiento. Cuando se emplee la monitorización de potenciales evocados motores es obligado el uso de protectores bucales para evitar una lesión de tejidos y la obstrucción del tubo orotraqueal.

\section{CRANEOTOMÍA DESPIERTA}

Las principales indicaciones de una craneotomía con el paciente despierto es la resección de tumores o malformaciones vasculares localizadas en áreas elocuentes, así como la estimulación cerebral profunda en pacientes con enfermedad de Parkinson o epilepsia ${ }^{(15)}$.

El paciente se posiciona frente al anestesiólogo y al neurofisiólogo para permitir una estrecha comunicación e interacción entre todos con el fin de realizar adecuadamente las diferentes pruebas y el mapeo transoperatorio; sin embargo, este tipo de procedimientos habitualmente demoran varias horas, por lo que se debe facilitar un acceso inmediato para asegurar la vía

\begin{tabular}{|c|c|c|}
\hline Dispositivo & Ventajas & Desventajas \\
\hline Laringoscopía directa & Menos entrenamiento & $\begin{array}{l}\text { Dificultad cuando hay inmovilización } \\
\text { Aún con inmovilización se produce movimiento de columna } \\
\text { vertebral }\end{array}$ \\
\hline Dispositivos supraglóticos & $\begin{array}{l}\text { Sigue formando parte del algoritmo de vía aérea en situacio- } \\
\text { nes en que no se puede intubar y no se puede ventilar }\end{array}$ & $\begin{array}{l}\text { La presión aplicada a las vértebras cervicales puede ser } \\
\text { suficiente para desplazar la columna }\end{array}$ \\
\hline Estilete luminoso & Menos movilización cervical & Técnica a ciegas \\
\hline Videolaringoscopio & $\begin{array}{l}\text { Mejor visualización de cuerdas vocales } \\
\text { Se requiere menor entrenamiento }\end{array}$ & $\begin{array}{l}\text { No siempre disponible } \\
\text { Sangre o secreciones pueden obstruir la cámara }\end{array}$ \\
\hline Fibrobroncoscopio & $\begin{array}{l}\text { Excelente para pacientes cooperadores } \\
\text { Permite examen neurológico posterior a intubación }\end{array}$ & $\begin{array}{l}\text { Técnicamente difícil } \\
\text { Capacitación específica } \\
\text { Tiempo prolongado de intubación } \\
\text { No apropiada para pacientes ansiosos }\end{array}$ \\
\hline
\end{tabular}

Modificada de: Austin N, et al(8). 
aérea en caso de alguna emergencia durante el transoperatorio.

El síndrome de apnea obstructiva del sueño debe ser considerado como criterio de exclusión para la craneotomía con paciente despierto debido al alto riesgo de obstrucción severa de la vía aérea.

En la técnica dormido-despierto-dormido, en la cual el manejo anestésico consiste en proporcionar anestesia general antes y después del mapeo cerebral, se ha reportado el uso de dispositivos supraglóticos como la técnica más aceptada. Uno de los momentos más críticos en la cirugía con el paciente despierto es justo antes del mapeo cerebral; mientras el paciente se posiciona en la mesa quirúrgica con el cabezal de Mayfield o con el marco de estereotaxia, es crucial evitar un reflejo tusígeno que pudiera resultar en lesión cervical o de escalpe ${ }^{(16)}$.

En el manejo de la vía aérea, el conocimiento del uso de mascarillas laríngeas es clave, ya que al ser flexibles tienen la posibilidad de acomodarse en la orofaringe en cualquiera de las diferentes posiciones extremas en las que se coloca al paciente para facilitar la craneotomía ${ }^{(17)}$.

Si se compara la intubación endotraqueal con los dispositivos supraglóticos, éstos ofrecen algunas ventajas, tales como evitar la extensión del cuello para la laringoscopía y su fácil posicionamiento. Las mascarillas laríngeas de segunda generación con canal para aspiración gástrica son las más recomendadas, ya que además permiten la inserción de un tubo de aspiración gástrica, reduciéndose así el riesgo de insuflación gástrica y regurgitación.

\section{CONCLUSIONES}

Como se ha dicho, la prioridad máxima del manejo de la vía aérea en este tipo de pacientes es evitar la manipulación agresiva; debe tomarse en cuenta llevar a cabo la inmovilización cervical oportuna y disminuir la posibilidad de producir lesiones secundarias por una movilización excesiva.

Habrá que recordar que la meta última del manejo adecuado de la vía aérea en general, y muy particularmente en estos casos, es evitar la hipoxia ${ }^{(18)}$.

A pesar de que cada vez los dispositivos supraglóticos reemplazan con mayor frecuencia la intubación endotraqueal en cirugía electiva, no existe hoy en día evidencia suficiente para probar que estos dispositivos de nueva generación proporcionen la misma seguridad que la intubación endotraqueal, contra la broncoaspiración.

Los anestesiólogos trabajamos para prevenir o minimizar las lesiones del sistema nervioso secundarias al manejo de la vía aérea, en presencia o no de lesiones cervicales; de esta forma, también contribuimos a mejorar los resultados de los diferentes procedimientos en el paciente neuroquirúrgico.

Es indispensable, por otra parte, tener el conocimiento de la patología que sufre el paciente; así como optimizar nuestras habilidades y saber emplear correctamente el equipo con el que se cuenta, para realizar una adecuada planeación del manejo integral, en beneficio de nuestros pacientes.

\section{REFERENCIAS}

1. Spiekermann BF, Stone DJ, Bogdonoff DL, Yemen TA. Airway management in neuroanaesthesia. Can J Anaesth. 1996;43:820-834.

2. López RSI, Díaz MM, Diez SY, González RGS. Abordaje de la vía aérea en la cirugía oncológica de cabeza y cuello. Revista Cubana de Anestesiología y Reanimación. 2013;12:139-150.

3. Gracia I, López AM, Valero R. Airway management in neurosurgical procedures (along with ventilation management). In: Khan $\mathrm{Z}$ (eds). Challenging topics in neuroanesthesia and neurocritical care. Springer, Cham. 2017. p. 109.

4. Oriol-López SA. Vía aérea difícil en neuroanestesia. Rev Mex Anest. 2006;29:169-175.

5. Fabregas N, Hurtado P, Gracia I, Craen R. Anestesia para neurocirugía mínimamente invasiva. Revista Colombiana de Anestesiología. 2015;43:15-21.

6. De la Serna-Soto HJ. Criterios de extubación temprana en el paciente neuroquirúrgico. Anestesia en México. 2018;30:41-47.

7. Schleicher P, Pingel A, Kandziora F. Safe management of acute cervical spine injuries. EFORT Open Rev. 2018;3:347-357.

8. Austin N, Krishnamoorthy V, Dagal A. Airway management in cervical spine injury. Int J Crit Illn Inj Sci. 2014;4:50-56.

9. Durga P, Sahu BP. Neurological deterioration during intubation in cervical spine disorders. Indian J Anaesth. 2014;58:684-692.

10. Theodore N, Hadley MN, Aarabi B, Dhall SS, Gelb DE, Hurlbert RJ, et al. Prehospital cervical spinal immobilization after trauma. Neurosurgery. 2013;72 Suppl 2:22-34.
11. Martínez-Ruiz YI, Vázquez-Torres J. Vía aérea en el paciente politraumatizado: utilidad de videolaringoscopía, como una alternativa y solución. Rev Mex Anest. 2017;40:113-119.

12. Bao FP, Zhang HG, Zhu SM. Anesthetic considerations for patients with acute cervical spinal cord injury. Neural Regen Res. 2017;12:499-504.

13. Apfelbaum JL, Hagberg CA, Caplan RA, Blitt CD, Connis RT, Nickinovich DG, et al. Practice guidelines for management of the difficult airway: an updated report by the American Society of Anesthesiologists Task Force on Management of the Difficult Airway. Anesthesiology. 2013;118:251-270.

14. Orozco-Ramírez SM, Hernández-Sánchez BM, Miranda-González A, De Alba-Salmerón AL. Técnica anestésica paciente dormido-despierto para craneotomía de tumores en áreas funcionales. Reporte de dos casos. Rev Mex Anest. 2017;40:312-319.

15. Chui J. Anesthesia for awake craniotomy: An update. Colomb J Anesthesiol. 2015;43:22-28.

16. Ramírez-Paesano C. Anestesia para craneotomía con paciente despierto. Rev Mex Anest. 2013;36:1-3.

17. Torrealba RV, González GR, Marquina SAJ. Uso de dispositivos supraglóticos para el manejo de la vía aérea. Zota TES. 2014;3:102-106.

18. Urtubia R, Escudero E, Esparza O, Gutiérrez JM. El aporte de las nuevas guías de la Sociedad de Vía Aérea Difícil (DAS). Rev Chil Anest. 2015;44:151-162. 